\title{
Jet quenching in the gluon plasma from Landau damping of soft modes
}

\author{
Dmitri Antonov* \\ Institut für Theoretische Physik, Universität Heidelberg, \\ Philosophenweg 19, D-69120 Heidelberg, Germany \\ E-mail: dima@tphys.uni-heidelberg.de
}

\section{Hans-Jürgen Pirner}

Institut für Theoretische Physik, Universität Heidelberg, Philosophenweg 19, D-69120 Heidelberg, Germany

E-mail: H.J.Pirneretphys.uni-heidelberg.de

\begin{abstract}
We discuss a model of jet quenching, in which a parton traversing the quark-gluon plasma loses its energy by interacting with hard thermal gluons through the exchanges by soft gluons. The hard gluons are modeled by the Hard Thermal Loop effective theory, the soft gluons by the chromomagnetic condensate, the interaction mechanism between the two is Landau damping of the soft gluons by the hard ones. Within such a model, we calculate the jet quenching parameter of a gluon in SU(3) Yang-Mills theory and find that, when the temperature varies from $T=T_{c}=270 \mathrm{MeV}$ to $T=900 \mathrm{MeV}$, the jet quenching parameter rises from $\hat{q}=0$ to approximately $1.8 \mathrm{GeV}^{2} / \mathrm{fm}$.
\end{abstract}

8th Conference Quark Confinement and the Hadron Spectrum September 1-6, 2008

Mainz. Germany

\footnotetext{
* Speaker.
} 
At RHIC, and even more LHC, energies, radiative energy loss is the dominant mechanism for the energy loss of a parton traversing the quark-gluon plasma. Its qualitative picture is as follows. During the gluon's formation time $\tau \sim \frac{\Delta E}{p_{\perp}^{2}}$, the parton propagates over the coherence length $z_{c}=\tau$ and accumulates the transverse momentum squared $p_{\perp}^{2} \sim Q^{2} \frac{z_{c}}{\lambda}$, where $\lambda$ is the mean free path, and $Q^{2}$ is $p_{\perp}^{2}$ transferred to the parton per one kick. Therefore, two scattering regimes are possible: $z_{c}<\lambda$, that corresponds to the incoherent scattering, and $z_{c} \sim L_{\|}>\lambda$, where $L_{\|}$is the distance travelled by the parton, that corresponds to the coherent scattering. In the second case, as follows from the formulae above, $\Delta E \sim \hat{q} L_{\|}^{2}$ (the Landau-Pomeranchuk-Migdal interference effect [1]), where $\hat{q} \sim \frac{Q^{2}}{\lambda}$ is the so-called jet quenching parameter. Due to its proportionality to $L_{\|}^{2}$, the radiative energy loss is much stronger than the collisional energy loss, due to whom $\Delta E \propto L_{\|}$only. More quantitatively [2],

$$
\Delta E=\frac{\alpha_{s}}{8} C_{R} \hat{q} L_{\|}^{2}, \quad \text { where } \quad \hat{q}=\rho\left\langle\sigma p_{\perp}^{2}\right\rangle .
$$

Here, $C_{R}$ is the quadratic Casimir operator of the representation $R$ of the parton and $\alpha_{s}$ is the strong coupling constant at an appropriate scale. In a dilute plasma [2],

$$
\hat{q} \propto T^{3} \int_{m_{D}^{2}} \frac{\mathrm{d} \sigma}{\mathrm{d} p_{\perp}^{2}} p_{\perp}^{2} \mathrm{~d}^{2} p_{\perp} \sim \alpha_{s}^{2} N_{c}^{2} T^{3} \ln \frac{1}{g}
$$

is determined by the density of scattering partners $\rho \propto T^{3}$, and $\mathrm{d} \sigma_{\text {Rutherford }} / \mathrm{d} p_{\perp}^{2} \sim p_{\perp}^{-4}$ is cut off in the IR at the Debye mass squared, $m_{D}(T)=g T \sqrt{N_{c} / 3}$ in $\mathrm{SU}\left(N_{c}\right)$ Yang-Mills theory.

In this talk, we summarize a recent nonperturbative calculation [3] of the jet quenching parameter. Our approach uses the dipole formalism [4], where a faked dipole of size $L_{\perp}$ is constructed from the partons in the $T$-amplitude and in the $T^{*}$-amplitude, the trajectories of which are displaced from each other by the distance $L_{\perp}$. The transport parameter $\left\langle\sigma p_{\perp}^{2}\right\rangle$ can then be calculated through the cross section of this faked dipole. It reads

$$
\left\langle\sigma p_{\perp}^{2}\right\rangle=\int \frac{\mathrm{d}^{2} p_{\perp}}{(2 \pi)^{2}} p_{\perp}^{2} \int \mathrm{d}^{2} L_{\perp} \mathrm{e}^{i \mathbf{p}_{\perp} \mathbf{L}_{\perp}} \int \mathrm{d}^{2} b\left\langle\operatorname{tr} V(\mathbf{b}) V^{\dagger}\left(\mathbf{b}+\mathbf{L}_{\perp}\right)\right\rangle
$$

where $V(\mathbf{b})$ is the Wilson line of a parton with the impact parameter $\mathbf{b}^{1}$. The real-valued part of the emerging Minkowskian Wilson loop $\left\langle W_{L_{\|} \times L_{\perp}}^{\text {Mink }}\right\rangle=\left\langle\operatorname{tr} V(\mathbf{b}) V^{\dagger}\left(\mathbf{b}+\mathbf{L}_{\perp}\right)\right\rangle$ defines the absorptive interaction between the faked dipole and the medium. It is related to the jet quenching parameter as

$$
\operatorname{Re}\left\langle W_{L_{\|} \times L_{\perp}}^{\mathrm{Mink}}\right\rangle=\exp \left(-\frac{\hat{q}}{4 \sqrt{2}} L_{\|} L_{\perp}^{2}\right) .
$$

A radical difference of Eq. (1) from the Minkowskian Wilson loop in the vacuum is its exponential fall-off instead of the non-absorptive behavior $\mathrm{e}^{i(\ldots)}$. In our scenario of jet quenching, the gluon plasma has two components: The chromo-magnetic condensate describes the soft component (which alone produces the $\mathrm{e}^{i(\ldots)}$-behavior), while the Hard Thermal Loop effective theory [5] describes the hard component. We argue that the exponential fall-off of Minkowskian Wilson loop

\footnotetext{
${ }^{1}$ Note that the corresponding formula of Ref. [3] has to be corrected in this way. This correction, however, does not affect the calculation performed in that paper.
} 
emerges due to interactions between the parton and the on-shell hard thermal gluons. These interactions are mediated by the soft gluons, which are Landau damped by the hard gluons.

We outline the main steps of our calculation. First, we consider the soft component of the gluon plasma, which we model through the stochastic vacuum model [6]. In Euclidean space-time at $T=0$, the Wilson loop in this model can be represented in terms of an effective local field theory of the field-strength tensor $F_{\mu \nu}^{a}$ as [3]

$$
\langle W(C)\rangle=\operatorname{tr} \int \mathscr{D} F_{\mu \nu}^{a} \mathrm{e}^{-S_{\text {Eucl }}[F]}
$$

with the action

$$
S_{\text {Eucl }}[F]=\frac{1}{2} \int \mathrm{d}^{4} x\left[F_{\mu \nu}^{a} \mathscr{K}(x) F_{\mu \nu}^{a}+i F_{\mu \nu}^{a} t^{a} \Sigma_{\mu v}\right] .
$$

Here $\Sigma_{\mu v}(x)=\int_{\Sigma(C)} \mathrm{d} \sigma_{\mu v}(w(\xi)) \delta(x-w(\xi))$ is the surface tensor, $\xi=\left(\xi_{1}, \xi_{2}\right), \xi_{1,2} \in[0,1], t^{a}$ is the $\mathrm{SU}\left(N_{c}\right)$-generator in the appropriate representation,

$$
\mathscr{K}(x)=\frac{N_{c}^{2}-1}{2 \pi^{2}} \frac{\mu^{4}}{\left\langle g^{2} F^{2}\right\rangle}\left(1-\frac{\partial^{2}}{\mu^{2}}\right)^{5 / 2},
$$

where $\left\langle g^{2} F^{2}\right\rangle$ is the gluon condensate, and $\mu^{-1}$ is the vacuum correlation length. Note that we disregard the anyhow small [7] contour $\times$ contour self-interactions of the Wilson loop, which do not lead to the expectation value of the form of Eq. (1).

At $T>T_{c}$, the correlator $\left\langle E_{i}^{a}(x) E_{k}^{b}\left(x^{\prime}\right)\right\rangle$ vanishes due to the deconfinement. Furthermore, according to the lattice data [8], the correlator $\left\langle E_{i}^{a}(x) B_{k}^{b}\left(x^{\prime}\right)\right\rangle$ can be neglected when compared to $\left\langle B_{i}^{a}(x) B_{k}^{b}\left(x^{\prime}\right)\right\rangle$. The latter can be parametrized as

$$
\left\langle g^{2} B_{i}^{a}(x) B_{k}^{b}\left(x^{\prime}\right)\right\rangle=\frac{\left\langle g^{2} F^{2}\right\rangle_{T}}{12\left(N_{c}^{2}-1\right)} \delta^{a b} \delta_{i k} \mathrm{e}^{-\mu(T)\left|x-x^{\prime}\right|} .
$$

Note that the chromo-magnetic gluon condensate $\left\langle g^{2} F^{2}\right\rangle_{T}$ and the correlation length of the chromomagnetic vacuum, $\mu^{-1}(T)$, are temperature-dependent quantities.

Due to the Euclidean $x_{4}$-periodicity at finite temperature, the contour $C=L_{\|} \times L_{\perp}$ effectively splits into pieces (strips), whose extensions along the $x_{3}$ - and $x_{4}$-axes are $\beta \equiv 1 / T$ (see Fig. 1). Equations (2)-(4) yield for the strip closest to the origin:

$$
\left\langle W_{1-\text { strip }}^{\text {Eucl }}\right\rangle=\operatorname{tr} \int \mathscr{D} B_{2}^{a} \mathrm{e}^{-S_{\text {Eucl }[B]},} \quad \text { where } \quad S_{\text {Eucl }}[B]=\int \mathrm{d}^{4} x\left(B_{2}^{a} \mathscr{K} B_{2}^{a}+i B_{2}^{a} t^{a} \Sigma_{13}\right),
$$

and the vector-function parametrizing the surface of the strip reads $w_{\mu}(\xi)=\beta \xi_{1} t_{\mu}+L_{\perp} \xi_{2} r_{\mu}$, where $t_{\mu}=(0,0,1,1), r_{\mu}=(1,0,0,0)$. The interaction between two strips separated by the distance $\beta k$, where $k$ is an integer, reads

$$
\chi_{k}=\frac{C_{R}}{4} \int \mathrm{d} \sigma_{13}(w) \int \mathrm{d} \sigma_{13}\left(w^{\prime}\right) \mathscr{K}^{-1}\left(w-w^{\prime}\right), \quad \text { where } \quad \mathscr{K}^{-1}(x)=\frac{\left\langle g^{2} F^{2}\right\rangle_{T}}{6\left(N_{c}^{2}-1\right)} \mathrm{e}^{-\mu(T)|x|}
$$

and $w_{\mu}^{\prime}=w_{\mu}\left(\xi^{\prime}\right)+(0,0, \beta k, 0)_{\mu}$. Accordingly, the overall contribution to the Wilson loop has the form

$$
-\ln \left\langle W_{L_{\|} \times L_{\perp}}^{\mathrm{Eucl}}\right\rangle=\sum_{i=0}^{n-1} \sum_{k=0}^{i} \chi_{k}=\sum_{k=0}^{n-1}(n-k) \chi_{k},
$$




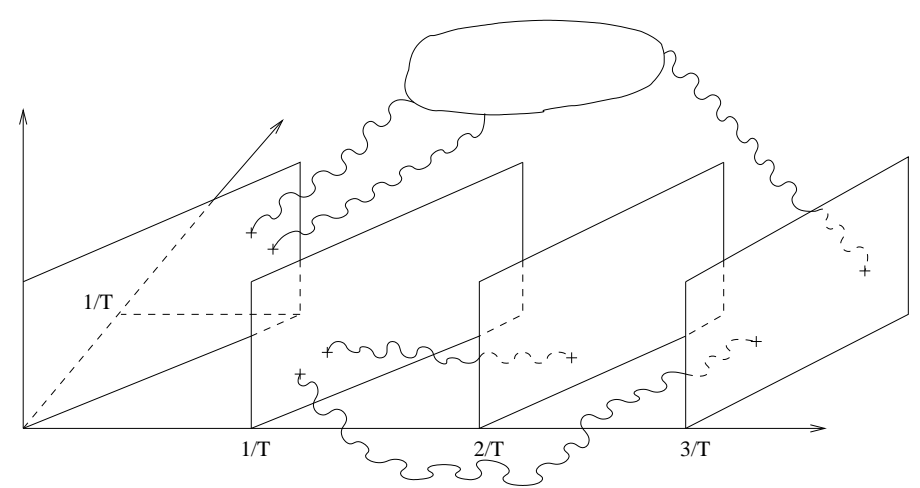

Figure 1: Depicted is the stack of strips representing the surface of the Wilson loop at finite temperature. The interactions between the strips are mediated by soft gluons. The closed loop represents the hard gluon. The mechanism of its interaction with the soft gluons is Landau damping.

where $n \equiv k_{\max }=L_{\|} /(\beta \sqrt{2})$ is the full number of strips.

We proceed now to the Minkowski space-time and account for the hard gluons from the heat bath, which appear as a polarization insertion into the $\left\langle B_{i}^{a}(x) B_{k}^{b}\left(x^{\prime}\right)\right\rangle$-correlator of soft gluons. By virtue of the Hard Thermal Loop effective theory [5], we can parametrize the polarization effects in the Minkowskian action of soft gluons as [3]

$$
S_{\text {Mink }}[B]=\int \mathrm{d}^{4} x\left\{B_{2}^{a}[\mathscr{K}(x)-i \mathscr{P}(x)] B_{2}^{a}+B_{2}^{a} t^{a} \Sigma_{13}\right\},
$$

where

$$
\mathscr{P}(p)=-\frac{M^{2}(T)}{p^{2}}, \quad M^{2}(T) \equiv \frac{\pi \zeta\left(1-\zeta^{2}\right)}{4} m_{D}^{2}(T), \quad \zeta \equiv \frac{\left|p_{0}\right|}{|\mathbf{p}|} \ll 1 .
$$

Using in Eqs. (5)-(7) $S_{\text {Mink }}[B]$ instead of $S_{\text {Eucl }}[B]$, we calculate the real-valued part of the Wilson loop, Eq. (1). This yields the desired jet quenching parameter in the form [3]

$$
\hat{q}=\frac{C_{R} \mathscr{N}(T) M^{2}(T)}{4 \pi^{2} n} \sum_{k=0}^{n-1}(n-k) \int_{-1}^{1} \frac{\mathrm{d} x}{\sqrt{k^{2}+2 k x+2 x^{2}}} \int_{0}^{\infty} \frac{\mathrm{d} p p^{4} J_{1}\left(p \beta \sqrt{k^{2}+2 k x+2 x^{2}}\right)}{p^{4}\left(p^{2}+\mu^{2}(T)\right)^{5}+\mathscr{N}(T) M^{4}(T)},
$$

where

$$
\mathscr{N}(T) \equiv\left(\frac{2 \pi^{2} \mu(T)\left\langle g^{2} F^{2}\right\rangle_{T}}{N_{c}^{2}-1}\right)^{2}
$$

and $J_{1}$ is the Bessel function. Equation (8) corresponds to the scattering of a fast parton off a hard thermal gluon in the leading approximation $\propto\left\langle g^{2} F^{2}\right\rangle_{T}^{2}$. The actual values of the jet quenching parameter can be obtained from Eq. (8) by subtracting $\hat{q}\left(T_{c}\right)$. That means we set $\hat{q}\left(T_{c}\right)=0$ since, due to the much lower density of scattering partners in the hadronic phase, typical values of $\hat{q}$ in that phase are by two orders of magnitude smaller than in the quark-gluon plasma [9].

We calculate $\hat{q}$ numerically for the case when the propagating parton is a gluon, and the theory under study is $\mathrm{SU}(3)$ pure Yang-Mills, where $T_{c}=270 \mathrm{MeV}$ [10]. We define the parameter $M(T)$ through the perturbative one-loop strong coupling [10]:

$$
g^{-2}(T)=2 b_{0} \ln \frac{T}{\Lambda}, \quad \text { where } \quad b_{0}=\left.\frac{11 N_{c}}{48 \pi^{2}}\right|_{N_{c}=3}=\frac{11}{16 \pi^{2}} \text { and } \Lambda \simeq 0.104 T_{c} .
$$


The temperature-dependent chromo-magnetic condensate can be obtained from the formula [7] $\left\langle g^{2} F^{2}\right\rangle_{T}=(72 / \pi) \mu^{2}(T) \sigma(T)$ with the same lattice data [10] suggesting the parametrizations $\sigma(T)=\left[0.566 g^{2}(T) T\right]^{2}$ and $\mu(T)=1.04 g^{2}(T) T$. The coefficient 1.04 in the last equation has been fixed by demanding the continuity with the low-temperature value [8]: $\mu\left(T_{c}\right)=894 \mathrm{MeV}$. Furthermore, we have checked that the numerical results for $\hat{q}$ are stable with respect to variations of the parameters $\zeta \ll 1$ and $n \gg 1$. For example, for $\zeta=0.1$ we find [3]

$$
\hat{q}(900 \mathrm{MeV})_{n=10}=1.78 \mathrm{GeV}^{2} / \mathrm{fm}, \quad \hat{q}(900 \mathrm{MeV})_{n=50}=1.98 \mathrm{GeV}^{2} / \mathrm{fm} .
$$

In the whole range of temperatures from $T_{c}$ to $900 \mathrm{MeV}$, a good fit to the numerical results is provided by the function

$$
\hat{q}(T)_{n=10}^{\mathrm{fit}}=0.26\left(T / T_{c}\right)^{3} \mathrm{GeV}^{2} / \mathrm{fm} .
$$

In conclusion, we note that our results have the same order of magnitude as those obtained by other nonperturbative methods, both in QCD and in $\mathscr{N}=4$ supersymmetric Yang-Mills theory. The detailed plots and comparison with other approaches can be found in Ref. [3].

\section{References}

[1] L. D. Landau and I. Pomeranchuk, Limits of applicability of the theory of bremsstrahlung electrons and pair production at high-energies, Dokl. Akad. Nauk Ser. Fiz. 92 (1953) 535; A. B. Migdal, Bremsstrahlung and pair production in condensed media at high-energies, Phys. Rev. 103 (1956) 1811.

[2] R. Baier, Yu. L. Dokshitzer, A. H. Mueller, S. Peigne and D. Schiff, Radiative energy loss of high energy quarks and gluons in a finite-volume quark-gluon plasma, Nucl. Phys. B 483 (1997) 291 [arXiv:hep-ph/9607355].

[3] D. Antonov and H.-J. Pirner, Jet quenching parameter $\hat{q}$ in the stochastic QCD vacuum with Landau damping, Eur. Phys. J. C 55 (2008) 439 [arXiv:0710.1540 [hep-ph]].

[4] J. Dolejsi, J. Hüfner and B. Z. Kopeliovich, Color screening, quark propagation in nuclear matter and the broadening of the momentum distribution of Drell-Yan pairs, Phys. Lett. B 312 (1993) 235 [arXiv:hep-ph/9305238].

[5] For a review see: J. P. Blaizot and E. Iancu, The quark-gluon plasma: Collective dynamics and hard thermal loops, Phys. Rept. 359 (2002) 355 [arXiv:hep-ph/0101103].

[6] For a review see: A. Di Giacomo, H. G. Dosch, V. I. Shevchenko and Yu. A. Simonov, Field correlators in QCD: Theory and applications, Phys. Rept. 372 (2002) 319 [arXiv:hep-ph/0007223].

[7] A. I. Shoshi, F. D. Steffen, H. G. Dosch and H. J. Pirner, Confining QCD strings, Casimir scaling, and a Euclidean approach to high-energy scattering, Phys. Rev. D 68 (2003) 074004 [arXiv:hep-ph/0211287].

[8] A. Di Giacomo, E. Meggiolaro and H. Panagopoulos, Gauge-invariant field strength correlations in QCD at zero and non-zero temperature, Nucl. Phys. B 483 (1997) 371 [arXiv:hep-lat/9603018].

[9] J. Casalderrey-Solana and X. N. Wang, Energy dependence of jet transport parameter and parton saturation in quark-gluon plasma, Phys. Rev. C 77 (2008) 024902 [arXiv:0705.1352 [hep-ph]].

[10] G. Boyd, J. Engels, F. Karsch, E. Laermann, C. Legeland, M. Lutgemeier and B. Petersson, Thermodynamics of SU(3) lattice gauge theory, Nucl. Phys. B 469 (1996) 419 [arXiv:hep-lat/9602007]. 